\title{
Molecule and dust reprocessing by the reverse shock in the supernova remnant Cas $A$
}

\author{
Chiara Biscaro \\ Basel University \\ E-mail: chiara.biscaro@unibas.ch \\ Isabelle Cherchneff \\ Basel University \\ E-mail: isabelle.cherchneffeunibas.ch
}

Dust and molecules are observed in various supernovae ( $\mathrm{SNe}$ ) and their remnants, but their formation and evolution in these hostile, shocked environments are still unclear. In some remnants, such as the 330 years-old SN remnant Cas A, the reverse shock (RS) is currently reprocessing the material formed after the SN explosion. Recently, transitions of warm CO have been detected with the Spitzer, AKARI and Herschel telescopes in Cas A ([9], [12]). In particular, CO lines were detected with Herschel in a small O-rich clump, and a high CO column density and temperature, compatible with shocked gas, were derived from line modelling ([12]). These observations thus show that a fair quantity of $\mathrm{CO}$ reforms after the passage of the RS. The Cas A remnant results from the explosion of a $19 \mathrm{M}_{\odot}$ star as a Type IIb supernova ([6]), characterised by a lowdensity ejecta. We first model the SN ejecta chemistry to identify the molecules and dust clusters that form after the explosion and are reprocessed by the RS. We find that Cas A progenitor could have formed large quantities of molecules and dust only in a dense ejecta involving clumps. We then model the impact of the RS on an oxygen-rich ejecta clump, considering various RS speeds and investigating the post-shock chemistry. We consider the destruction of molecules and dust clusters by the shock and their reformation using a chemical kinetic model. The impact of UV photons coming from the hot post-shock region on the ionization fraction of the post-shock gas is included. We also model the sputtering (thermal and non-thermal) of the dust by the RS. We found that the reverse shock destroys the molecules and clusters present in the O-rich clump. CO reforms in the post shock gas with abundances that concur with the latest Herschel observations, confirming a post-shock origin for the submm CO lines. We then derive a dust size distribution for the ejecta of the Cas A progenitor, and investigate the effect of different RS velocities on this dust size distribution. Our results show that medium- and large-sized grains can survive the RS and that small dust clusters do not efficiently reform in the shocked gas. This result indicates that the dust formed in the SN ejecta and destroyed by the RS is unable to reform from the gas phase in the SN remnant.

The Life Cycle of Dust in the Universe: Observations, Theory, and Laboratory Experiments 18-22 November, 2013

Taipei, Taiwan 


\section{Model and method}

The chemistry of the gas phase is modelled using a chemical kinetic description ([3], [10]). The chemical network consists of 90 chemical species and over 400 reactions, including thermolecular formation and fragmentation, neutral-neutral reactions, charge exchange, radiative association and destruction by Compton electrons. In the chemistry of the postshock gas, destruction by energetic UV photons is also taken into account. The nucleation of silicate clusters of enstatite and forsterite is described according to the nucleation route given by [5] and [10], where the growth to enstatite $\left(\mathrm{MgSiO}_{3}\right)$ and forsterite $\left(\mathrm{Mg}_{2} \mathrm{SiO}_{4}\right)$ dimers take place through successive oxidation process by $\mathrm{O}_{2}$ and $\mathrm{SO}$ and addition of atomic $\mathrm{Mg}$. We consider two temperature regimes in the ejecta: the "hot T ejecta" (6600-300 K) from 100 up to 1000 days after explosion, and the "cool T ejecta" (300-50 K) from 1000 to 3000 days. After modelling the ejecta phase, we concentrate on one single, dense, O-rich knot and we consider the impact of the RS on the clump with shock velocities that range between 35 to $200 \mathrm{~km} \mathrm{~s}^{-1}$ ([2], [4]). We model the reformation of molecules and we consider the possibility of reforming dust clusters in the cooling post-shock gas. We also investigate the effect of the reverse shock on the sputtering of dust. The main sputtering atom in an oxygen-rich knot is $\mathrm{O}^{+}$, and we calculated the thermal and non-thermal sputtering on forsterite grains ([11], [7]). A size distribution is derived for these dust grains, for ejecta densities of $\sim 10^{11}$ $\mathrm{cm}^{-3}$ (typical of a type IIP), and the effect of sputtering is investigated for a shock velocitiy of 200 $\mathrm{km} \mathrm{s}^{-1}$.

\section{Results}

\subsection{Ejecta chemistry}

The low density ejecta of Cas A leads to the formation of molecules but dust clusters do not form in large amounts. (Figure 1, left). This is in contrast with the Spitzer data on dust in Cas A ([9]).
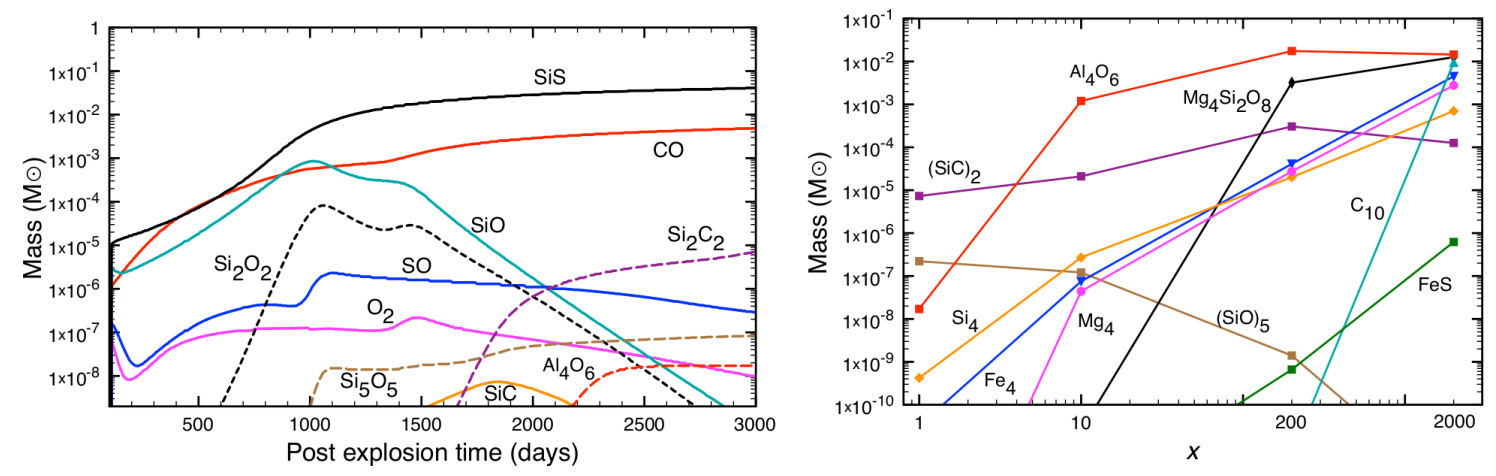

Figure 1: Left: mass of molecules and clusters in the Cas A supernova ejecta. Right: cluster masses at 3000 days, as a function of increment in density (x) with respect to the standard case.

Figure 1 (right) shows that gas density increments with respect to the Type IIb case leads to more chemically complex dust clusters. This implies a clumpy ejecta, with high-density gas knots and density contrast $>100$ (see [1] for more detail). 


\subsection{Post-shock chemistry}

We look at the passage of the reverse shock in a single, high density, oxygen rich clump. We consider a different range of possible shock speeds (Table 1) for the gas-phase chemistry. For the $200 \mathrm{~km} \mathrm{~s}^{-1}$ shock molecules quickly reform after the gas starts to cool in the photoionised region (PIR), but with abundances 1000 times smaller than in the ejecta.

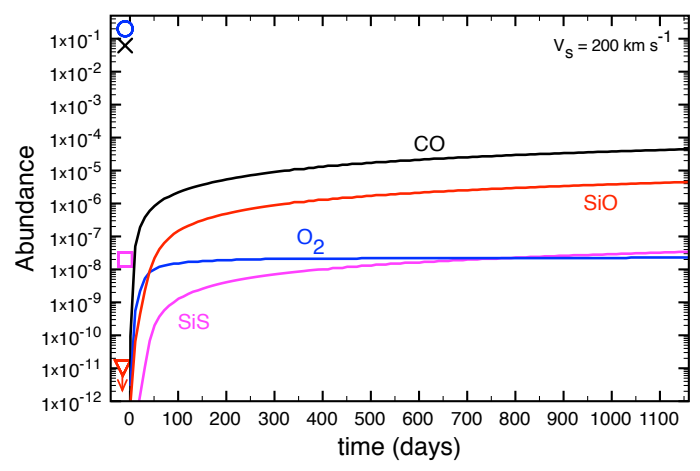

Figure 2: Abundances of main molecules in the PIR (with respect to total gas number density) for a $200 \mathrm{~km} \mathrm{~s}^{-1}$ shock. Simbols indicate abundances in the pre-shock gas at 3000 days post-explosion for $\mathrm{O}_{2}$ (open circle), $\mathrm{CO}$ (cross), $\mathrm{SiS}$ (open rectangle) and $\mathrm{SiO}$ (triangle with arrow). The $\mathrm{SiO}$ pre-shock abundance is $10^{-17}$ ([1]).

Slower shocks lead to lower densities and shorter PIR and less molecules reform (Figure 3). Dust clusters are not able to reform for any shock speed, even for increased post-shock gas densities, and their abundance range is between $10^{-20}$ and $10^{-7}$ (see [1] for more detail).
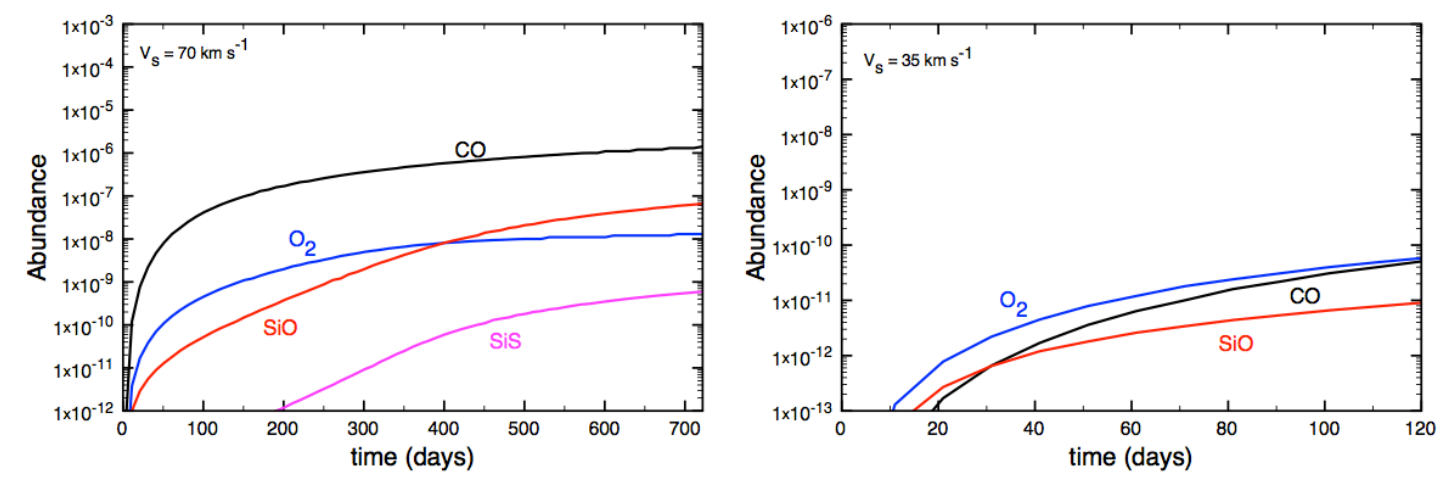

Figure 3: Abundances in the PIR for $70 \mathrm{~km} \mathrm{~s}^{-1}$ (left) and $35 \mathrm{~km} \mathrm{~s}^{-1}$ (right) shock

\subsection{Dust formation and sputtering}

We took the forsterite grain pre-shock size distributions (Sarangi, priv.comm.) and applied sputtering by oxygen ions for a $200 \mathrm{~km} \mathrm{~s}^{-1}$ shock crossing a clump with an overdensity factor of 200 with respect to normal ejecta. The pre-shock and post-shock grain size distributions are plotted in Figure 4. While the smallest grains are destroyed and returned to the gas phase, most of the medium- and large-sized grains survive the passage of the reverse shock. 


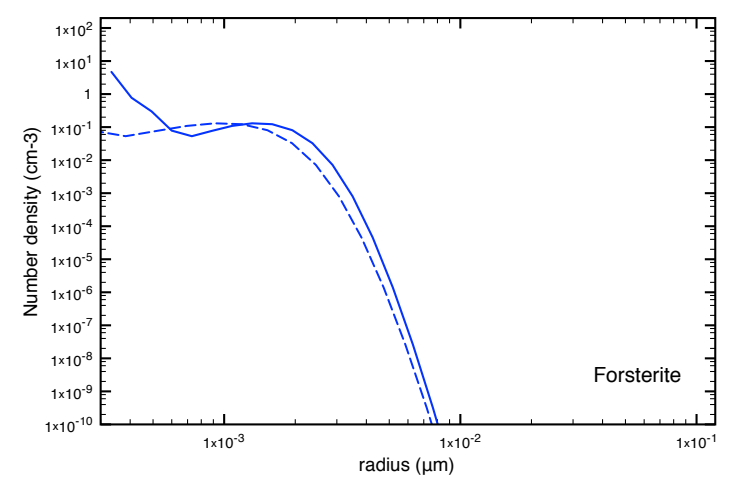

Figure 4: Full line: pre-shock grain size distribution at 4000 days post-explosion in a clump with an overdensity factor of 200. Dashed line: grain size distribution 1200 days after the passage of the $200 \mathrm{~km} \mathrm{~s}^{-1}$ reverse shock

\section{References}

[1] Biscaro, C. \& Cherchneff, I., Molecules and dust in Cassiopeia A. I. Synthesis in the supernova phase and processing by the reverse shock in the clumpy remnant, 2014, A\& A, 546,25

[2] Borkowski, K. \& Shull, J.M., Pure-oxygen radiative shocks with electron thermal conduction, 1990, ApJ, 348, 169

[3] Cherchneff, I. \& Dwek, E. ,The Chemistry of Population III Supernova Ejecta. I. Formation of Molecules in the Early Universe, 2009, ApJ, 703, 642

[4] Docenko, D. \& Sunyaev, R.A., Fine-structure infrared lines from the Cassiopeia A knots, 2010, A\& A, 509, A59

[5] Goumans, T.P.M., Bromley, S.T., Efficient nucleation of stardust silicates via heteromolecular homogeneous condensation, 2012, MNRAS, 420, 3344

[6] Krause, O., Birkmann, S., Usuda, T. et al., The Cassiopeia A Supernova Was of Type IIb, 2008, Science, 320, 1195

[7] Nozawa T., et al., Dust Destruction in the High-Velocity Shocks Driven by Supernovae in the Early Universe, 2006, ApJ, 648, 435

[8] Rauscher T., Heger A., Hoffman R.D., Woosley S.E., Nucleosynthesis in Massive Stars with Improved Nuclear and Stellar Physics, 2002, ApJ, 576, 323

[9] Rho J., et al., Freshly Formed Dust in the Cassiopeia A Supernova Remnant as Revealed by the Spitzer Space Telescope, 2008, ApJ, 673, 271

[10] Sarangi, A. \& Cherchneff, I., The Chemically Controlled Synthesis of Dust in Type II-P Supernovae, 2013, ApJ, 776, 107

[11] Tielens, A.G.G.M., et al., The physics of grain-grain collisions and gas-grain sputtering in interstellar shocks, 1994, ApJ, 431, 321

[12] Wallström, S. H. J.; Biscaro, C.; Salgado, F., et al., CO rotational line emission from a dense knot in Cassiopeia A. Evidence for active post-reverse-shock chemistry, A \& A, 2013, 558, L2 